\title{
Procesos de gestión del talento humano en el sector educativo gerencial de Colombia
}

\section{Processes of human talent management in the management educational sector of Colombia}

\author{
Ruby Borrero \\ rubistherforero@gmail.com \\ Institución Educativa Distrital Gabriela Mistral \\ Colombia \\ https://orcid.org/0000-0003-4326-9176
}

Recibido: 30 de octubre del 2018

Aprobado: 01 de diciembre del 2018

\section{RESUMEN}

El presente trabajo es un estudio analítico bibliográfico, que tiene como objetivo analizar los procesos de gestión del talento humano en el sector educativo gerencial de Colombia. Sustentado bajo teorías de: Chiavenato (2011), Franklin y Krieger (2012), Tobon (2009), entre otros. Enmarcado bajo un paradigma racional, en cuanto al diseño el mismo se fundamentó en un trabajo bibliográfico. Cuando se habla del talento humano se hace referencia a personas con habilidades y capacidades. Gestionar adecuadamente el talento humano permitirá a una organización crecer y consolidarse en el mercado, por esto, el área encargada de llevar a cabo esta tarea es de vital importancia dentro de una organización. La finalidad de los procesos de gestión del talento humano actualmente en las organizaciones ha trascendido de forma vertiginosa a medida que en los procesos de toma de decisiones define un liderazgo a la vanguardia y notablemente capacitado en aptitudes y actitudes. Es importante el desarrollo de este trabajo porque contribuirá al conocimiento de uno de los factores más álgidos y trascendentales de la administración, brindando nuevos enfoques para el buen desenvolvimiento, de tal manera que se logren los objetivos trazados, especialmente, en el ámbito administrativo

Descriptores: Procesos; gestión; talento humano; organización; sector educativo. 


\section{ABSTRACT}

The present work is a bibliographic analytical study, which aims to analyze the processes of human talent management in the educational management sector of Colombia. Sustained under the theories of: Chiavenato (2011), Franklin and Krieger (2012), Tobon (2009), among others. Framed under a rational paradigm, in terms of design it was based on a bibliographic work. When talking about human talent, reference is made to people with skills and abilities. Proper management of human talent will allow an organization to grow and consolidate in the market, therefore, the area in charge of carrying out this task is of vital importance within an organization. The purpose of human talent management processes currently in organizations has transcended vertiginously as decision-making processes define a leadership at the forefront and remarkably trained in aptitudes and attitudes. The development of this work is important because it will contribute to the knowledge of one of the most critical and transcendental factors of the administration, providing new approaches for good development, in such a way that the objectives set, especially in the administrative sphere, are achieved.

Descriptors: Processes; management; human talent; organization; education sector.

\section{INTRODUCCIÓN}

A través de la historia de la humanidad, el ser humano se ha agrupado en comunidades para poder sumar sus esfuerzos y afrontar los requerimientos que le plantea el entorno. Es a través de la asociación, comunicación y cooperación que han surgido distintos modelos de organización social en los cuales la suma de los esfuerzos, persigue objetivos que resulta imposible lograr con iniciativas individuales.

Dentro de este marco de referencia, como evolución natural, surge entonces la creación de un sistema de patrones de comportamiento, expresiones, prácticas comunes y en definitivas normas, reglas y por supuesto las leyes que, enmarcado en un ámbito territorial definido, da forma a las naciones y sus correspondientes formas de gobierno. Por tanto, ya desde una concepción moderna, los estados han volcado su atención en los mecanismos bajo los cuales desempeñan sus funciones demostrando un creciente interés por la forma en que realizan su gestión. 
En este contexto, la reforma del Estado y la gestión del talento humano, se han convertido en el tema central de la agenda política mundial. De allí que, en el mundo de la postguerra, se hizo necesario que las voluntades políticas manifestadas en los organismos multilaterales tuviesen un impacto en la gobernanza de las naciones, haciendo hincapié en una serie de criterios mínimos bajo los cuales pueda medirse el desempeño de los gobiernos como una forma de garantizar una estabilidad política a nivel mundial.

Asimismo, es importante destacar que, en muchas organizaciones, la denominación de administración de recursos humanos se está sustituyendo por gestión del talento humano, gestión de socios, gestión de colaboradores, gestión de capital humano, administración del capital intelectual e incluso gestión de personas entre otras.

Sin embargo, estos cambios de denominación llevan consigo un espacio y una configuración nueva del área de recursos humanos, reflejando una manera de tratar a las personas; así las organizaciones sustituyen la concepción de personal, trabajadores, recursos humanos, por el de socios de la organización, en la cual las personas invierten su talento, tiempo, dedicación y habilidades en las empresas donde laboran.

Es así como las organizaciones exitosas, descubren en el valor y las habilidades de las personas que están en ella, su principal ventaja competitiva, por ello, tratan a sus miembros como socios de la empresa y no como simples empleados, trabajadores 0 recursos productivos de la organización. Al tratar las organizaciones a las personas como recursos, deben ser administrados, lo cual implica planeación, organización, dirección y control de sus actividades y por lo tanto sujetos pasivos de la acción organizacional.

En cambio, al ser tratadas como socios, las personas deben ser gestionadas para alcanzar el éxito personal y organizacional, de tal forma que cada uno de las partes alcance sus objetivos y cumpla su misión. En este caso Chiavenato (2011), se refiere a la gestión del talento humano como la función que permite la colaboración eficaz de las 
personas (empleados, funcionarios, recursos humanos o cualquier denominación utilizada) para alcanzar los objetivos organizacionales e individuales.

En ese sentido entonces, para llevar a cabo su función, los gerentes necesitan satisfacer objetivos múltiples. Es necesario hacer frente a necesidades sociales, de la organización y personales. Estos objetivos se pueden lograr mediante actividades diversas de personal, enfocadas a mantener, utilizar, evaluar y conservar una fuerza laboral eficaz.

Por tanto, es tarea del gerente gestionar el talento humano de la organización, es así como, cada administrador, director, gerente, supervisor, desempeña en su trabajo las cuatro funciones administrativas a saber: planear, organizar, dirigir y controlar. A esto no se escapa, los procesos de gestión del talento humano, pues están relacionados con las funciones del administrador, refiriéndose a las políticas y prácticas necesarias para administrar el trabajo con las personas: diseño de cargo, reclutamiento y selección, orientación, remuneración, comunicación, higiene, entre otras.

Con referencia a lo expuesto en el párrafo anterior, se busca entonces que las personas se conviertan en elementos esenciales para las organizaciones, aprovechando los esfuerzos del trabajo en conjunto para alcanzar las estrategias organizacionales, dejando de lado los interés personales y apuntado a los objetivos grupales, todo esto implica tener expectativas positivas, respeto a los demás, intentando comprender a los otros para generar y mantener un buen clima laboral, a través del dinamismo, la energía, inteligencia, creatividad y racionalidad para lograr ser más competitivo, cabe resaltar, que los trabajadores son el talento humano más importante que tienen las organizaciones.

De acuerdo a lo expuesto anteriormente, se hace conveniente entender como es el desempeño de los procesos de gestión del talento humano, enfocándose fundamentalmente en los organismos educativos gerenciales. Es evidente que son distintos los factores que se ven involucrados en el proceso de gestión del talento 
humano que se ve influenciado por el accionar del personal adscrito a las dependencias, así como el control ejercido sobre estas.

\section{FUNDAMENTACIÓN TEÓRICA}

Para el desarrollo de los fundamentos teóricos de esta investigación, se consideró conveniente estudiar una serie de aspectos relevantes a los procesos de gestión del talento humano, por lo que se hace necesario establecer claramente la correspondencia entre algunos conceptos referidos a la variable; con el fin de construir sustentos teóricos que le de concreción a estos términos, los cuales son indispensables para una mejor comprensión de la misma:

\section{Gestión del talento humano}

Dada la naturaleza, es impensable concebir un modelo óptimo de gestión, sin involucrar la dimensión humana de las organizaciones. Esto conduce irremediablemente a analizar el proceso de gestión del talento humano en las organizaciones colombianas, y muy particularmente a aquellas que forman parte del presente estudio. Para ello se presentan los distintos preceptos teóricos, que rigen el estudio de la gestión del talento humano.

Por ello entonces según Franklin y Krieger (2012) toda organización está integrada por individuos que constituyen su capital humano de quienes depende el buen funcionamiento y el logro de los objetivos. Para los autores la gestión del talento humano en las organizaciones abarca muchas y variadas tareas relacionadas con los vínculos laborales, presentes entre los individuos y con la organización en general. Destacan entre estas, la búsqueda y selección de los futuros integrantes, el desarrollo de sus capacidades y la evaluación de su desempeño, con todas las actividades que cada una de estas funciones generales representa.

De la misma manera, el DAFP (2012) establece que la gestión del talento humano es aquella orientada al desarrollo y cualificación de los servidores públicos buscando la 
observancia del principio de mérito para la provisión de los empleos, el desarrollo de competencias, vocación del servicio, la aplicación de estímulos enfocados a la consecución de resultados. Esto, es establecido para todos los entes en Colombia, indistintamente del nivel o ámbito territorial al cual este adscrito el organismo.

En el mismo orden de ideas, según el DPN (2007, p.35) la gestión del talento humano pretende la ejecución de una serie de procesos que garantizan que se cumplan con los servicios establecidos para lo cual se debe vincular, o contratar, el recurso humano idóneo, capacitado y necesario para el cumplimiento de las diferentes funciones. Otros aspectos, están relacionados con la administración del régimen salarial y prestacional del personal, la capacitación y el desarrollo del personal, así como la aplicación del régimen disciplinario, fiscal y penal.

Es de entender que, en el ámbito educativo gerencial de Colombia, se deba acatar las disposiciones legales y normativas establecidas, las cuales de forma genérica pretenden apoyarse en una adecuada gestión del talento humano para el logro de los objetivos establecidos. No obstante, dichas normativas nacen de los modelos teóricos clásicos, y frecuentemente se observa la adaptación de las teorías aplicables propias de la administración de los recursos humanos como una forma de perseguir la uniformidad y coherencia en la gestión.

\section{Procesos de gestión del talento humano}

Al momento de abordar los procesos de gestión del capital humano, es importante conocer el concepto de proceso. Dessler (2015) afirma que el proceso es un conjunto de etapas enlazadas las cuales se conjugan con un objetivo particular, las cuales transforman elementos de entrada en resultados. Los procesos de gestión incluyen el Reclutamiento, Selección de personal, Capacitación y Evaluación del desempeño.

Por otra parte, según Louart (2015) comprende un proceso al conjunto de acciones o actividades sistematizadas que se realizan o tienen lugar con un fin determinado, aplicándose en el ámbito organizacional a la obtención de determinados resultados 
como producto de esfuerzos específicos. Los procesos de gestión del capital humano se sub dividen en fases que son consecutivas, incluyendo el Reclutamiento, Selección de personal, Capacitación y Evaluación del desempeño.

De acuerdo con Villegas (2016) sostiene que la gestión del capital humano comprende las actividades focalizadas en lograr la configuración de un personal con características idóneas para los cargos diseñados. Para ello, las empresas o instituciones diseñan procesos de reclutamiento, selección, inducción, capacitación y evaluación focalizados en detectar las características del personal e incluir a aquel con las mejores competencias.

Al respecto, Alles (2009) agrega que las organizaciones cuentan con diversidad de procesos, cada uno de los cuales están vinculadas las etapas focalizadas en unos objetivos. Estos procesos se ejecutan a través de actividades programadas usualmente en forma secuencial, con lo cual es posible establecer la sucesión de eventos entre el reclutamiento y la selección final de los candidatos. Estos procesos permiten alcanzar los objetivos de gestión propuestos por las organizaciones.

\section{Proceso para la integración del personal}

Para, Münch (2011), la integración es el proceso a través del cual se convoca, elige e introduce a las personas más adecuadas de acuerdo con los requerimientos de la organización. En este sentido, las organizaciones resuelven sus necesidades de recursos humanos, entre ellas el pronóstico de sus necesidades futuras, el reclutamiento y selección de candidatos y la inducción del personal de nuevo ingreso Igualmente, Gómez (2010) señala que el proceso de integración del personal es la contratación de trabajadores, incluye también la colaboración para que el personal de nuevo ingreso se adapte fácilmente a la organización, moverse ágilmente en ella (rotación de puestos) y salir de la empresa. Es también el proceso en la cual el personal empieza a comprender y aceptar los valores, normas de la empresa.

De acuerdo con lo que plantea Chiavenato (2011), representan la ruta que conduce a 
su ingreso en la institución. Es la puerta de entrada que solo se abre para los candidatos que tienen características y competencias personales que se ajustan a las que predominan en ella. El momento en que los elementos humanos se integran a una institución tiene mucha importancia y, por lo mismo, debe ser vigilado con especial cuidado.

\section{Procesos para la recompensa del personal}

Según, Chiavenato (2011), constituyen los elementos fundamentales para incentivar y motivar al personal de la institución, siempre que los objetivos organizacionales sean alcanzados y los objetivos individuales sean satisfechos. Por tal razón los procesos para ofrecer recompensas destacan entre los principales procesos administrativos del área de recursos humanos dentro de las organizaciones. La palabra recompensa significa una retribución, premio o reconocimiento por los servicios de alguien.

Igualmente, Chávez (2011), manifiesta que la recompensa suele ser un incentivo ofrecido para la realización de una determinada acción. En este sentido, el concepto del proceso para la recompensa del personal está asociado a un premio o un estímulo, por la labor desempeñada con los alumnos y con el personal administrativo de la institución, todo esto en pro del bien de la misma.

\section{Procesos para el desarrollo del personal}

Chiavenato (2011), plantea que desarrollar a las personas no significa únicamente proporcionarle información para que aprendan nuevos conocimientos, habilidades y destrezas y, así, sean más eficientes en lo que hacen. Significa, sobre todo, brindarle la información básica para que aprendan nuevas actitudes, soluciones, ideas y conceptos y para que modifique sus hábitos, comportamientos y sean más eficaces en lo que hacen.

Por otro lado, González (2010), define el desarrollo de personas, capacidad para emprender acciones eficaces para mejorar el talento y las capacidades de los demás. 
Este desarrollo permite que el docente afronte desafíos significativos en el ámbito educativo, puesto que son espacios dinámicos donde cada miembro de la institución educativa debe poner en práctica toda su habilidad y destreza para culminar el proceso asignado.

Por su parte, Tobon (2009), expone que son "las competencias fundamentales para vivir y desenvolverse en la sociedad en cualquier ámbito laboral. Dichas competencias están caracterizadas por: 1 . Constituir la base sobre la cual se forman los demás tipos de competencia; 2 . Se forman en la educación básica y media; 3 . analizan, comprenden y resuelven problemas de la vida cotidiana; 4 . Constituyen un eje central en el proceso de la información".

En síntesis, estos métodos permiten alcanzar los objetivos de gestión propuestos por las organizaciones lo cual constituye un reto en el ámbito organizacional, por cuanto se destacan como parte fundamental en la búsqueda y selección de los futuros integrantes, el desarrollo de sus capacidades y la evaluación de su desempeño, con todas las actividades que cada una de estas funciones generales representa, contribuyendo de esta manera al mejoramiento de los procesos de gestión del talento humano que a su vez reflejará sus resultados en el aumento del estándar de calidad de vida de sus empleados y el desarrollo de su organización.

\section{METODOLOGÍA}

Al instaurar la metodología a manejar para el proceso de una investigación, debe tomarse en cuenta los distintos elementos del contexto humana y social, ya que son los elementos que van a fijar el uso de las distintas técnicas comunes, que habrán de utilizarse para considerar los aspectos metodológicos que requiere la misma. Igualmente, para contextualizar el conocimiento de manera clara, se necesita fundamentar de forma epistemológica la investigación, es por ellos que, es obligatorio dar respuesta a una cadena de incógnitas que esclarezcan el proceso investigativo. 
Por ello, un modelo epistemológico o cognitivo compone un conjunto de creencias, normas y operaciones que precisan cómo hay que crear ciencia; son los modelos de gestión para la búsqueda del conocimiento, destacándose para ese producto distintos enfoques epistémicos que según Bunge (1972, p. 13), "la epistemología, es la rama de la filosofía que estudia la investigación científica y su producto, el conocimiento científico".

En ese sentido, puede decirse que la epistemología conoce de las dificultades filosóficas que envuelven la proposición del conocimiento, es la ciencia que experimenta el conocimiento humano y la forma en que los individuos operan para ampliar su ordenación de pensamiento. Sumado a estas afirmaciones Tamayo y Tamayo (2012, p. 23), agrega que "la epistemología representa ciencia o teoría de la ciencia y tiene por esencia conocer los sucesos por su condición y en sus causas".

Ahora bien, lo que busca la epistemología es expresar complicaciones tales como: la naturaleza y la producción de las doctrinas científicas (conceptos, hipótesis, modelos, teorías, etc.), la correspondencia de cada una de ellas con la realidad, cómo la ciencia refiere, expone, pronostica y ayuda al control de la naturaleza (esto último en conjunto con la filosofía de la tecnología), la formulación y uso del método científico, los prototipos de consideraciones utilizadas para producir conclusiones y los alcances de los distinto métodos y modelos de ciencia.

Así pues, puede expresarse que el paradigma del presente estudio quedó situado como racional, el cual es concebido por Padrón (2008), como aquel que es provocado por métodos de razonamiento, pero que además no admite sentimientos ni afectos. En este sentido, se motiva sobre las ideas de la enseñanza y del aprendizaje de manera objetiva.

De igual forma, cuyo propósito general será analizar los procesos de Gestión del talento humano en el sector educativo gerencial de Colombia, desde esta perspectiva se ubica dentro del tipo analítica en este sentido, Hurtado (2010) la define como aquella que busca razonar los escenarios en términos de las relaciones de sus componentes. 
Pretende revelar los elementos que forman cada totalidad y las interconexiones que da cuenta de su integración.

Asimismo, la investigación de tipo analítica tiene como finalidad razonar un suceso y entenderlo en términos de sus semblantes menos evidentes, circunscribiendo tanto el análisis como la síntesis. Analizar representa disgregar o alterar un conjunto en todas sus partes. Síntesis significa congregar distintas cosas de manera que formen una totalidad coherente, dentro de un juicio más amplio del que se tenía al inicio, así lo refiere Padrón (2008).

En ese sentido, los aspectos metodológicos de este estudio son tomados desde la comprensión holística, con soporte en la metodología ofrecidas por diferentes autores, soportándose en la sistematización de los diseños señalados en la referente propuesta, donde el diseño responde a la categoría del fin que se intenta lograr y al nivel de conocimiento a crear con la investigación.

Ahora bien, en cuanto al diseño la misma se fundamentó en una investigación bibliográfica, la cual es definida por Tamayo y Tamayo (2012, p.113) como "aquellas investigaciones a las que se apela al manejo de información o datos alcanzados por otros, alcanzando al investigador de manera ya realizada de acuerdo a los objetivos de quienes primeramente lo ejecutaron y manipularon". Del mismo modo, Arias (2012) lo establece como un proceso fundamentado en la indagación, rescate, examen, crítica e interpretación de antecedentes secundarios, es decir, los conseguidos y asentados por otros estudiosos en fuentes documentales; impresas, audiovisuales o electrónicas.

Según, Perdomo (2007), el diseño bibliográfico recolecta la información, primariamente, de libros revistas, periódico, entre otros. El investigador hace referencia de las fuentes primarias y secundarias de datos, la primera se fundamenta en alegatos escritos por individuos que han vivido el acontecimiento explicado, la segunda son escritos de personas que describen afirmaciones de otras personas sobre algún suceso.

La recolección de la información es de gran relevancia para la investigación, estas técnicas se seleccionara dependiendo del tipo y diseño de la investigación. Sobre las 
fichas de trabajo o fichaje, comenta Perdomo (2007) que acopian la información bibliográfica, es decir, aquellas que se recoge de libros, periódicos y demás documentos.

Cabe recalcar que, como lo menciona Nava (2008), el fichaje ha cambiado a través de los años motivados por los progresos tecnológicos. Los materiales de la técnica están compuestos por las fichas $u$ hojas sueltas y debido a las innovaciones tecnológicas, a través del método de computación, el cual fue el utilizado en el presente estudio.

Ahora bien, sobre la observación establece Tamayo y Tamayo (2012) que es la técnica más habitual en una investigación ya que la observación indica y causa los problemas y conlleva a la necesidad de la sistematización de información. Igualmente hace mención a la observación indirecta, precisando esta como la observación realizada por el investigador de los datos recogidos por otros, ya sea de demostraciones orales 0 escritas.

Esta última, podría concebirse como observación documental la cual es definida por Bodington (2011, p. 138) "como la observación directa de documentos, en ella se descubre, consigue, consulta, extrae, compila y elige la información necesaria para la disertación o investigación de fuentes escritas". La presente investigación cuenta con un tipo de investigación analítica y diseño bibliográfico, por ende las técnicas de recolección utilizadas son la del fichaje y observación documental antes descritas.

\section{HALLAZGOS REFLEXIVOS DE LA INVESTIGACIÓN}

Seguidamente, se presentan los hallazgos obtenidos en los resultados concernientesa un proceso reflexivo que se genero a lo largo del recorrido del estudio:

1. Antiguamente en las empresas se denominaba área de recursos humanos, pero en la actualidad se la asigna el nombre de gestión de talento humano. Esto innova la imagen y el concepto de medir el equipo de profesionales que deseamos para lograr el cometido de la organización. 
2. Ciertamente, las empresas deben considerar este campo de vital importancia para lograr a través de su personal calificado alcanzar las metas y objetivos propuestos, por ende, deben estimular al trabajador brindándoles las armas para innovar o en todo caso otorgar facilidades. Todo ello favorecerá al éxito de la organización sea cual fuera su misión.

3. El principal objetivo de la gestión del talento humano es el alcance de las metas trazadas por la organización, por ello, es fundamental garantizar la eficacia y el máximo desarrollo del recurso humano, es decir, asegurar que esta cuente con colaboradores calificados y motivados.

4. Es vital que los colaboradores tengan en claro e internalicen cuáles son los objetivos de la organización. Es importante también, crear un equilibrio entre las metas individuales y las corporativas.

5. La gestión del talento humano es importante porque es el socio estratégico de las demás áreas, pues se encarga de potenciar a los colaboradores, el trabajo en equipo, teniendo como resultado el éxito de la organización.

6. El sistema técnico solo es posible gracias a las personas, pues están son las encargadas de su funcionamiento a fin de que haya una correcta medición de las tareas realizadas y objetivos alcanzados.

7. Debemos concluir que la mayor concentración de un alto ejecutivo actualmente está en la atención y compromiso del recurso humano a fin de lograr y ayudar a la empresa en sus objetivos estratégicos.

8. Es fundamental resaltar el papel importante que desempeñan los gerentes de las organizaciones, ya que ellos son los principales líderes en ejecutar o diseñar los procesos para la mejora continúa de las empresas.

\section{CONSIDERACIONES FINALES}

Tomado en consideración todo lo anterior se concluye que; las organizaciones se desarrollan en tamaño y complejidad y la administración en consecuencia enfrenta 
mayores dificultades, por la necesidad de controlar el rumbo de la empresa para el logro de los objetivos estratégicos y de negocios que se tienen planeados. $Y$ es en éste contexto donde se pone de manifiesto la vital importancia del Talento Humano, pues se podrá contar con las mejores estrategias, tecnología de punta y el capital necesario.

Pero finalmente son las personas los medios por los cuales se pueden materializar los objetivos estratégicos, y entonces no será suficiente tampoco ser empresas socialmente responsables, ni contar con líderes transformacionales es necesario y de vital importancia también la comunicación, que en cierta medida puede fungir como un eje al mismo tiempo que se interrelacionen estos factores para elevar a las organizaciones a un mejor desempeño y esto solo será posible con estrategias de gestión del Recurso Humano para así mejorar las condiciones de la empresa. Al respecto García; García e Isea (2017) señalan que:

Se debe transformar la cultura del trabajo, la producción y distribución de la riqueza, incrementar la calidad de vida, recomponer el tejido social y la cohesión, para construir un proyecto en el que participen todos. Asumir el hecho de que en los procesos gerenciales convergen disímiles pensamientos, diferentes experiencias, que requieren ser conjugados desde la transdisciplinariedad. (p. 87)

\section{REFERENCIAS CONSULTADAS}

1. Alles, M. (2009). Diccionario de Comportamientos. Gestión por competencias. Granica. Buenos Aires. Argentina.

2. Arias, E. (2012). El Proyecto de Investigación. Introducción a la Metodología Científica. Editorial Episteme. 4ta. Edición. Caracas, Venezuela.

3. Bodington, M. (2011). Metodología de la investigación jurídica. Fondo Editorial URBE.

4. Bunge, M (1972) La investigación científica: su estrategia y filosofía. Habana. Ciencias Sociales.

5. Chávez, N (2011). Introducción a la Investigación Educativa. Maracaibo: Editorial La Columna.

6. Chiavenato, H. (2011) Administración de recursos humanos El capital humano de las organizaciones. McGraw-Hill interamericana editores, S.A. México.

7. Departamento Administrativo de la Función Pública (2012) Plan nacional de formación y capacitación de empleados públicos para el desarrollo de 
competencias. Tercera Edición. Ediciones de la escuela superior de administración pública. Bogotá Colombia.

8. Departamento Nacional de Planeación (2007) Gestión Pública Local. Imprenta Nacional de Colombia. Bogotá Colombia.

9. Dessler, G. (2015) Administración de recursos humanos. Decimocuarta edición. Pearson educación. México.

10.Franklin, E. y Krieger, M. (2012) Comportamiento Organizacional. Pearson Educación. México.

11.García, L., García, J., \& Isea, J. (2017). Aportes de la nueva ciencia al pensamiento científico en el área gerencial. Revista Arbitrada Interdisciplinaria Koinonía, 1(1), 76-87. Recuperado de http://fundacionkoinonia.com.ve/ojs/index.php/revistakoinonia/article/view/16

12. Gómez, M (2010) Calidad de vida laboral en empleados temporales del Valle de Aburrá - Colombia. Revista Ciencias Estratégicas, vol. 18, núm. 24.

13.González, J. (2010) Formación emprendedora en el contexto de la universidad centro occidental Lisandro Alvarado, "revista científica digital del centro de investigación y estudios gerenciales" Venezuela. Pág. 18-22.

14. Hurtado, J. (2010). Metodología de la Investigación Holística, Caracas: SYPAL.

15.Louart, P (2015) Gestión de los Recursos Humanos. Ediciones Gestión. Barcelona. España.

16. Münch, L (2011). Fundamento de administración. México. Editorial Trillas. Pp. 235.

17.Nava (2008). Proceso y Producto de la Investigación Documental. $2^{\circ}$ Edición. EDILUZ. Maracaibo. Venezuela.

18.Padrón, J. (2008). Análisis del discurso e investigación social: temas para seminario. Publicado por el Decanato de Postgrado de la Universidad Nacional Experimental Simón Rodríguez. Venezuela.

19.Perdomo, R. (2007). Metodología de la investigación jurídica. Universidad de Los Andes, Consejo de publicaciones. EE.UU.

20. Tamayo y Tamayo, M. (2012). Metodología de la Investigación Científica. México: Editorial Trillas.

21.Tobón, S. (2009). Formación basada en competencias: Pensamiento complejo, diseño curricular y didáctica. Bogotá: ECOE.

22. Villegas, G. V. (2016). Predictores de la capacidad de Innovación en las organizaciones. Revisión sistemática de literatura. Espacios, 37(9): 3. 